\title{
Programa de exercícios físicos para o doente renal crônico em hemodiálise
}

\author{
Exercise program for patients with chronic kidney disease on hemodialysis \\ therapy
}

\author{
Moane Marchesan ${ }^{1}$ \\ Airton José Rombaldi ${ }^{2,3}$ \\ 1. Programa de Pós-Graduação em \\ Educação Física - Universidade Federal \\ de Santa Catarina, Florianópolis, SC, \\ Brasil. \\ 2. Programa de Pós-Graduação em \\ Educação Física - Universidade Federal \\ de Pelotas, RS, Brasil. \\ 3. GEEAF - Grupo de Estudos em \\ Epidemiologia da Atividade Física - \\ UFPel, Pelotas, RS, Brasil.
}

\begin{abstract}
Resumo
O número de pacientes em hemodiálise vem aumentando cada vez mais, fazendo-nos repensar sobre estratégias que possam ser efetivadas para auxiliar na reabilitação e na melhora da qualidade de vida dos mesmos. Uma estratégia que pode ser inserida no cotidiano desses pacientes é a prática regular de exercícios físicos, desde que sejam tomados alguns cuidados necessários para ocasionar benefícios à saúde dos mesmos. Este artigo descreve um programa de exercícios físicos para pacientes em hemodiálise que foi desenvolvido na Clínica Renal do Hospital Santa Lúcia, (Cruz Alta-RS), mostrando a atuação e a importância do profissional de Educação Física na unidade renal. Os dados evidenciados neste estudo sugerem que é possível desenvolver um programa de exercícios físicos para pacientes em hemodiálise, trazendo benefícios biopsicossociais para esta população.
\end{abstract}

Palavras-chave: Atividade motora; Hemodiálise; Doença renal crônica; Falha renal crônica.

\section{Abstract}

The number of hemodialysis patients has grown, leading researchers to focus on strategies that can be effective in assisting rehabilitation and improvement of quality of life among them. One of the strategies that can be added to their daily living is physical activity, as long as safety precautions are taken and benefits are assured. This article describes a physical exercises program for hemodialysis patients, which was developed at the Renal Clinic of the Santa Lucia Hospital (CRUZALTA-RS), showing the role and importance of physical education professional in the renal care service. The data shown in this study suggest that it is possible to develop an exercise program for patients on hemodialysis, resulting in biopsychosocial benefits for this population.

Keywords: Motor activity; Hemodialysis; Chronic kidney disease; Chronic renal failure.

\section{ENDEREÇOPARACORRESPONDÊNCIA}

\section{Moane Marchesan}

Av. Almirante Tamandaré, 748

Apto. 131, Bloco C

Bairro Coqueiros - Florianópolis, $\mathrm{SC}$ 88080-160

e-mail: moedfisio@yahoo.com.br

$\begin{array}{ll}\text { - Recebido: } & 14 / 11 / 2011 \\ \text { - Aceito: } & 07 / 12 / 2011\end{array}$




\section{INTRODUÇÃO}

A doença renal crônica (DRC) é caracterizada por uma lesão renal acompanhada de perda progressiva e irreversível da função dos rins, onde, em sua fase mais avançada, o organismo não consegue manter o equilíbrio do meio interno'. Nesta fase, classificada como fase 5 ou terminal ${ }^{2}$, o paciente necessita de uma terapia renal substitutiva (TRS) para manter a normalidade do organismo.

A forma mais utilizada de TRS de acordo com o Relatório Brasileiro de Diálise de $2010^{3}$ é a hemodiálise (HD), representando um total de $90,3 \%$. Os pacientes submetidos a HD apresentam um conjunto de sinais e sintomas denominado uremia, caracterizado por manifestações sistêmicas, fazendo com que, não somente os rins, mas todos os sistemas, passem a funcionar de maneira anormal ${ }^{4}$.

Um desses sinais é a baixa capacidade de praticar exercício $^{5}$ que, pode ser explicada pela atrofia, fraqueza e câimbras musculares ${ }^{6}$, como também pela redução da capacidade aeróbia7.

O exercício físico pode amenizar muitas consequências da $\mathrm{DRC}^{8}$, proporcionando benefícios como a melhora do controle pressórico ${ }^{9}$, a melhora da capacidade funcional ${ }^{10}$, meIhora da função muscular ${ }^{11}$, além da melhora da qualidade de vida ${ }^{12}$.

Apesar da literatura evidenciar os benefícios que o exercício físico pode trazer para pacientes em HD, poucos são os estudos de intervenção no Brasil, e poucas são as unidades de
HD que oferecem essa prática ao paciente.

Desta forma, este artigo tem por objetivo relatar um programa de exercícios físicos para o paciente em HD, ressaltando a importância do mesmo para a reabilitação dessa população, além de mostrar que a unidade renal pode ser um novo campo de trabalho para profissionais de Educação Física.

\section{O PROGRAMA}

Este programa foi desenvolvido na Clínica Renal do Hospital Santa Lúcia, na cidade de Cruz Alta - RS. No ano da realização do estudo a clínica atendia 72 pacientes na unidade de HD, todos pelo Sistema Único de Saúde.

Na figura 1 é possível analisar a estrutura do programa (modelo lógico).

A execução dos exercícios foi desenvolvida durante a sessão de HD, cuidando para não ultrapassar as duas primeiras horas, pois de acordo com Moore et al. ${ }^{13}$, após esse momento, os pacientes apresentam redução na pressão arterial e no débito cardíaco, inibindo a capacidade do exercício físico.

O programa seguiu as recomendações de Casaburi ${ }^{14}$, onde o autor propõe que os melhores resultados da prática de exercícios para pacientes em HD são os que têm frequência de três vezes na semana, e duração de 45-60 minutos.

O programa foi executado na seguinte sequência: alongamento antes mesmo de iniciar a sessão de HD, onde os pacientes foram orientados a permanecer em posição estática, durante o tempo pré-determinado (10-60 segundos); aque-

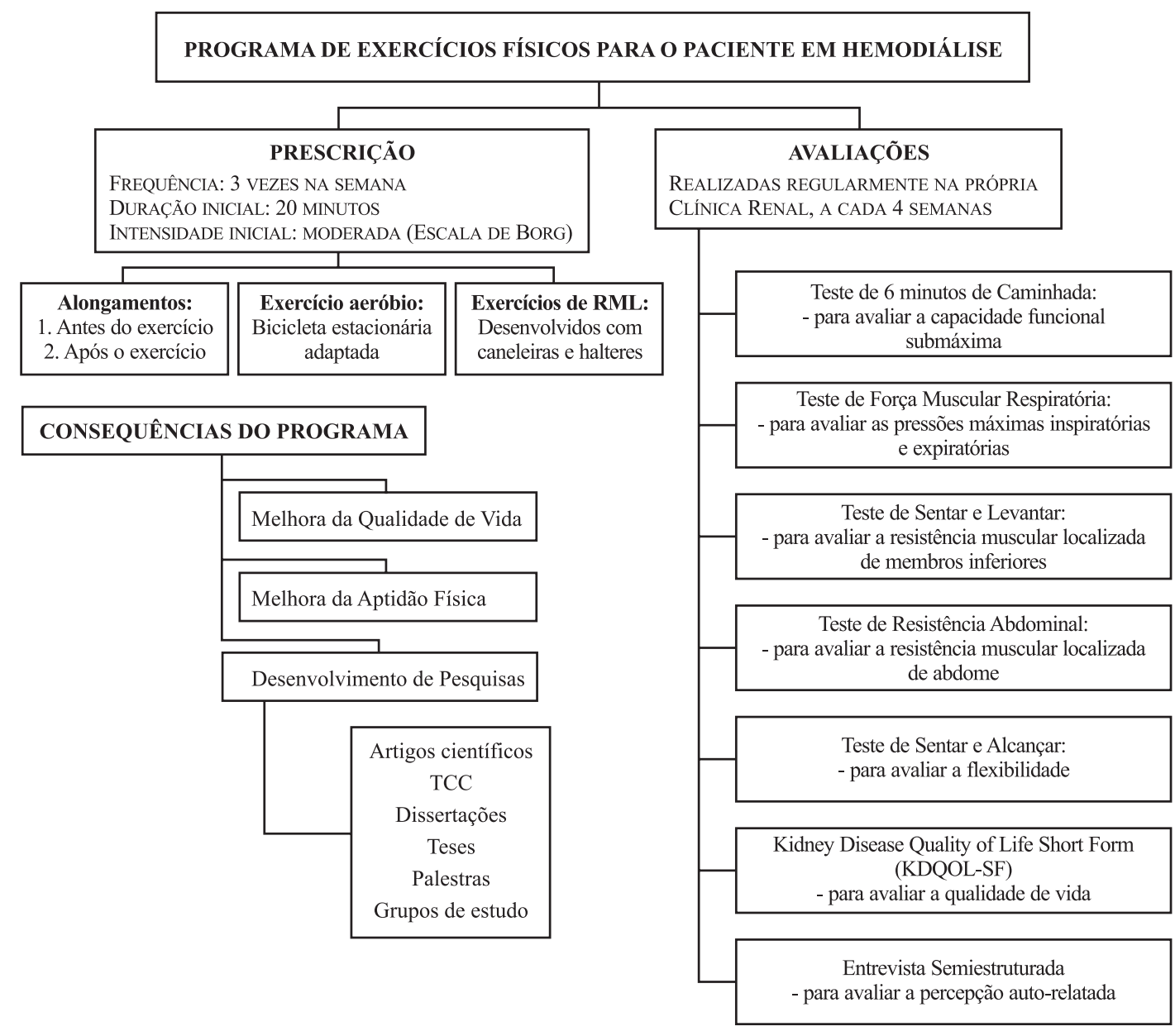


cimento, realizado na bicicleta estacionária sem carga, com duração de 3 minutos; treinamento aeróbio ainda na bicicleta estacionária, com duração inicial de 15 minutos, aumentando gradativamente; exercícios de resistência muscular localizada $(\mathrm{RML})$, iniciando sem carga, aumentando progressivamente; e alongamento passivo, realizado pelo profissional de Educação Física, para todos os segmentos corporais, exceto o braço da fístula.

Na figura 2 está exposta a prescrição dos exercícios.

Os exercícios de RML foram realizados para membros superiores e membros inferiores a partir dos seguintes exercícios: rosca direta, tríceps testa, elevação lateral, crucifixo, remada, abdominal, extensão de joelho, flexão de quadril, adução e abdução de quadril, dorsiflexão e flexão plantar do pé. Também foram realizados exercícios para os músculos respiratórios, com uso do manovacuômetro.

Os pacientes foram avaliados antes, durante e após o programa de exercícios físicos. Primeiramente, foi criada uma ficha diagnóstica, com dados do prontuário médico (idade, cor da pele, gênero, fator etiológico da DRC, o dia de início do tratamento hemodialítico, medicações e o número de co-morbidades apresentadas). Esses dados permitiram o melhor entendimento sobre os pacientes que foram submetidos ao programa de exercícios físicos.

A segunda avaliação foi constituída por uma bateria de testes físicos, para avaliar a aptidão física dos pacientes. Foram utilizados os seguintes testes:

- teste de seis minutos de caminhada: é um teste seguro, válido, confiável e, não necessita de muitos recursos para sua aplicação ${ }^{15}$. É indicado para avaliação de intervenções, bem como para predizer a capacidade funcional ${ }^{16}$. Ele foi realizado no corredor da clínica renal, seguindo as recomendações da American Thoracic Society ${ }^{17}$. Durante a realização do teste, os pacientes fizeram uso de um oxímetro de pulso, e tiveram monitorados também a frequência cardíaca, a pressão arterial e o nível de dispneia, de acordo com metodologia proposta por BORG, conforme indica a normatização brasileira ${ }^{15}$. Para a análise do teste de caminhada utilizou-se as equações propostas por Enright e Sherrill ${ }^{18}$

- teste de força muscular respiratória: para avaliar a pressões máximas inspiratória e expiratória. Para realização do teste utilizou-se um manovacuômetro (Famabras, Indústria Brasileira) escalonado em -300 a $+300 \mathrm{cmH}_{2} \mathrm{O}$, de acordo com a metodologia proposta por Black e Hyatt ${ }^{19}$. Os pacientes permaneceram na posição sentada, fazendo uso de um clipe nasal. Os mesmos foram instruídos a realizar três inspirações e expirações máximas, sendo considerada a média entre elas. Usou-se as equações propostas por Neder et $\mathrm{a}^{20}$ para predizer os valores.

- teste de sentar e levantar em 30 segundos: foi utilizado para avaliar a resistência muscular de membros inferiores, sugerido por Jones e Rikli21. O paciente foi orientado a sentar-se em uma cadeira com $45 \mathrm{~cm}$ de altura, com os pés apoiados no solo e com as costas eretas apoiadas no encosto da cadeira, os braços permaneceram contra o peito, ao nível dos punhos. Ao sinal de partida o paciente deveria levantar-se até ficar em pé regressando à posição sentada em um intervalo de 30 seg. Registrou-se o número máximo de repetições no tempo estipulado.

- teste de resistência muscular abdominal: para avaliar a resistência muscular de abdome. Para realização deste teste utilizou-se o protocolo do Manual Eurofit para Adultos ${ }^{22}$, onde o paciente permanece em decúbito dorsal, com os joelhos flexionados em aproximadamente $45^{\circ}$ e pés apoiados no chão. Ele é constituído por três níveis distintos: 1) os braços devem estar estendidos e as palmas das mãos devem estar sobre os músculos das coxas; 2) os braços devem ser cruzados junto ao peito, fazendo com que as mãos toquem os ombros contrários; 3 ) as pontas dos dedos das mãos deverão estar apoiadas nas orelhas. O avaliado deverá realizar cinco abdominais em cada nível para poder passar par o próximo. $\mathrm{O}$ avaliador deverá contar o número de repetições (0-15) em cada nível para poder classificar a resistência muscular.

- teste de sentar e alcançar, proposto por Wells e Dillon ${ }^{23}$ : foi utilizado para avaliar a flexibilidade, sendo realizado com auxílio do banco de Wells e Dillon, da marca cardiomed, com

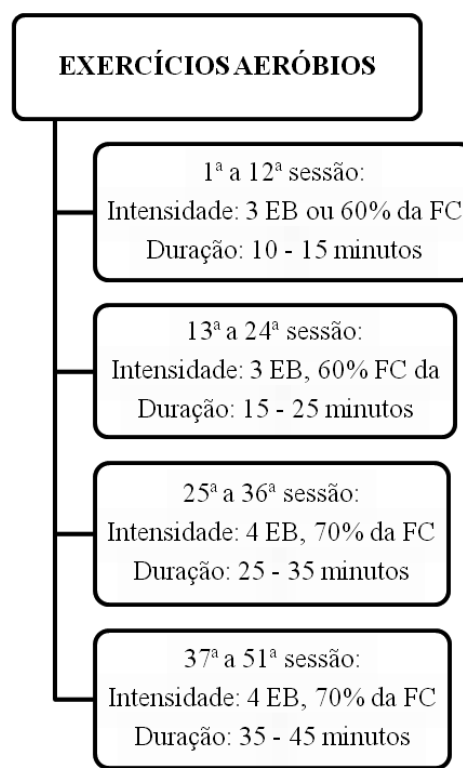

EXERCÍCIOS DE RML

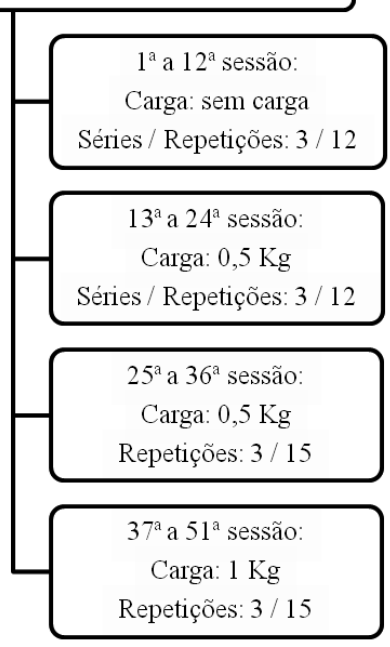

EB- Escala de BORG

$\mathrm{FC}=$ Frequência Cardíaca 
precisão de $0,1 \mathrm{~cm}$. Para a realização do teste, o banco foi colocado no solo e foi apoiado na parede, permitindo que o sujeito, ao sentar com as pernas estendidas, apoiasse os pés na madeira da caixa. Os pacientes permaneceram sentados, com os pés descalços, apoiados na caixa, sem flexionar os joelhos. Eles deveriam inclinar-se para frente, com as palmas das mãos para baixo e paralelas, até o máximo de sua flexão. A distância máxima alcançada foi registrada em centímetros, como medida de flexibilidade. Foram realizadas três tentativas, sendo anotada a melhor.

A terceira avaliação foi constituída pelo questionário Kidney Disease Quality of Life Short Form (KDQOL-SF), para avaliar a qualidade de vida. Este instrumento é específico para pacientes que estão em tratamento de diálise, é de fácil entendimento e preenchimento, apresenta validade ao objetivo que se propõe e já está traduzido para o português ${ }^{24}$. Este questionário foi aplicado antes e após o programa.

A última avaliação foi uma entrevista semiestruturada, realizada somente após o programa de exercícios físicos, onde os pacientes puderam relatar sobre a percepção que eles tiveram sobre o programa de exercícios físicos e os efeitos do mesmo na sua saúde.

\section{CONSIDERAÇÕES FINAIS}

Este programa de exercícios físicos trouxe benefícios para a reabilitação dos pacientes uma vez que contribuiu com a melhora da aptidão física e da qualidade de vida dos mesmos. Esses ganhos foram identificados no desempenho nos testes físicos, bem como nos relatos das entrevistas, onde os pacientes puderam se posicionar com relação à participação no programa.

Cabe ressaltar que além da prescrição e avaliação de exercícios físicos, o profissional de educação física deve deter-se em ensinamentos que os pacientes possam levar para a sua vida, compreendendo melhor a sua condição física e entendendo a importância de se manter ativo para ter uma qualidade de vida relacionada à saúde mais acentuada.

A inserção do profissional da Educação Física na unidade renal contribuiu também com o desenvolvimento de pesquisas, onde o envolvimento multidisciplinar acarretou em elaborações de trabalhos de conclusão de curso, dissertações de mestrado, tese de doutorado e publicações de artigos. A partir desse envolvimento também surgiram grupos de estudo e até mesmo um projeto de extensão, proporcionando aos alunos do curso de Educação Física a vivência hospitalar.

\section{Contribuições dos autores}

Moane Marchesan e Airton José Rombaldi contribuíram para todo o processo que envolveu o estudo, desde sua a formulação até a parte escrita do manuscrito.

\section{REFERÊNCIAS}

1. Romão Júnior JE. Doença Renal Crônica: definição, epidemiologia e classificação. J Bras Nefrol 2004;26:1-3.

2. Lewey AS, Coresh J, Balk E et al. National Kidney Foundation Practice Guidelines for Chronic Kidney Disease: Evaluation, Classification, and Stratification. Ann Intern Med 2003;139:137-47.

3. Sesso, Lopes AA, Thomé FS. Relatório do censo brasileiro de diálise de 2010. J Bras Nefrol 2011;33:442-47.

4. Barros E, Manfro RC, Thomé FS, Gonçalves LF. Nefrologia: Rotinas, Diagnósticos e Tratamento. Porto Alegre: Artes Médicas Sul, 2006.

5. Casaburi R. Treinamento de exercício reabilitativo em pacientes submetidos à diálise. In: Kopple; Massry. Cuidados Nutricionais Das Doenças Renais. Rio de Janeiro: Guanabara Koogan, 2004.

6. Floyd M, Ayyar DR, Barwick DD et al. Myopathy em chronic renal failure. QJM 1974;43:509-24.

7. Moore GE, Pearson DB, Stray-Gundersen et al. Uremic myopathy limts aerobic capacity in hemodialysis patients. Am J Kidney Dis 1993;22:277-87.

8. Smart N, Steele M. Exercise training in haemodialysis patients: a systematic review and meta-analysis. Nephrology 2011;16:626-32.

9. Miller BW, Cress CL, Jonhson ME, et al. Exercise duringhemodialysis decreases the use of antihypertensive medications. Am J Kidney Dis 2002;39:828-33.

10. Koufaki P, Naish PF, Mercer TH. Assessing the efficacy of exercise training inpatients with chronic disease. Med Sci Sports Exerc 2002;34:1234-41.

11. Headley S, Germain M, Mailloux P, Mulhern J, Ashworth B, Burris J, et al. Resistance training improves strength and functional measures in patients with end stage renal disease. Am J Kidney Dis 2002;40:355-64.

12. Painter P, Carlson L, Carey S, Paul SM, Myll J. Physical functioning and health related quality-of-life changes with exercise training in hemodialysis patients. Am J Kidney Dis 2000;35:482-92.

13. Moore GE, Painter PL, Brinker KR, et al. Cardiovascular response to submaximal stationary cycling during hemodialysis. J Kidney Dis 1998;31: 631-637.

14. Casaburi R. Treinamento de Exercício Reabilitativo em Pacientes Submetidos à Diálise. In: Kopple JD, Massry, SG. Cuidados Nutricionais Das Doenças Renais. Rio de Janeiro: Guanabara Koogan, 2004, 547-62.

15. Britto RR, Souza LAP. Teste de caminhada de seis minutos - uma normatização brasileira. Fisioter Mov 2001;19:49-54.

16. Solway $S$, Brooks D, Lacasse $Y$ et al. A qualitative systematic overview of the measurement properties of functional walk tests used in the cardiorespiratoy domain. Chest 2001;119:256-70.

17. ATS statement: guidelines for the six-minute walk test. Am J Respir Crit Care Med 2002;166:111-7.

18. Enright PL, Sherrill DL. Reference equations for the six-minute walk in healthy adults. Am J Respir Crit Care Med 1998;158:1384-7.

19. Black LF, Hyatt RE. Maximal respiratory pressures: normal values and relationship to age and sex. Am Rev Respir Dis 1969;99(5):696-702.

20. Neder J, Andreoni S, Lerario MC, et al. Reference values for lung function tests II. Maximal respiratory pressures and voluntary ventilation. Braz J Med Biol Res 1999;32:719-27.

21. Jones CJ, Rikli RE, Beam WC. A 30-s chair-stand test as a measure of lower body strength in community-residing older adults. Res Q Exerc Sport 1999;70:113-9.

22. Engstrom V. Aptitud músculo-esquelética. In: Oja PTuxworth B. Manual Eurofit para adultos: test Europeo de aptitud física. Tampere, 1995.

23. Wells KF, Dillon EK. The sit and reach: a test of back leg flexibility. Research quarterly. 1952;23.

24. Duarte OS, Miyazaki MCOS, Ciconelli MR, et al. Tradução e adaptação cultural do instrumento de avaliação de qualidade de vida para pacientes renais crônicos (KDQOL-SF). Rev Assoc Med Bras 2003;49:375-81. 